\title{
Evolution of mechanism of parton energy loss with transverse momentum at RHIC and LHC in relativistic collision of heavy nuclei
}

\author{
Dinesh K. Srivastava \\ Variable Energy Cyclotron Centre, 1/AF Bidhan Nagar, Kolkata 700064, India
}

\begin{abstract}
.
We analyze the suppression of particle production at large transverse momenta in $(0-5 \%$ most $)$ central collisions of gold nuclei at $\sqrt{s_{\mathrm{NN}}}=200 \mathrm{GeV}$ and lead nuclei at $\sqrt{{ }^{s} \mathrm{NN}}=2.76 \mathrm{TeV}$. Full next-to-leading order radiative corrections at $\mathcal{O}\left(\alpha_{s}^{3}\right)$, and nuclear effects like shadowing and parton energy loss are included. The parton energy loss is implemented in a simple multiple scattering model, where the partons lose an energy $\epsilon=\lambda \times d E / d x$ per collision, where $\lambda$ is their mean free path. We take $\epsilon=\kappa E$ for a treatment which is suggestive of the Bethe Heitler $(\mathrm{BH})$ mechanism of incoherent scatterings, $\epsilon=\sqrt{\alpha E}$ for LPM mechanism, and $\epsilon=$ constant for a mechanism which suggests that the rate of energy loss $(d E / d x)$ of the partons is proportional to total path length $(L)$ of the parton in the plasma, as the formation time of the radiated gluon becomes much larger than $L$. We find that while the $\mathrm{BH}$ mechanism describes the nuclear modification factor $R_{\mathrm{AA}}$ for $p_{T} \leq 5 \mathrm{GeV} / c$ (especially at RHIC energy), the LPM and more so the constant $d E / d x$ mechanism provides a good description at larger $p_{T}$. This confirms the earlier expectation that the energy loss mechanism for partons changes from BH to LPM for $p_{T} \geq \lambda<k_{T}^{2}>$, where $\lambda \approx 1 \mathrm{fm}$ and $<k_{T}^{2}>\approx 1$ $\mathrm{GeV}^{2}$ is the average transverse kick-squared received by the parton per collision. The energy loss per collision at the $\sqrt{{ }^{s} \mathrm{NN}}=2.76 \mathrm{TeV}$ is found to be about twice of that at $0.2 \mathrm{TeV}$.
\end{abstract}

\section{Introduction}

The suppressed production of particles having large transverse momenta, known as jet-quenching [1, 2] along with the strong collective flow [3, 4] and the success of recombination model [5] in explaining the constituent quark number scaling observed for the elliptic flow, at the experiments performed at the Relativistic Heavy-Ion Collider (RHIC) at Brookhaven National Laboratory have provided a strong evidence for the production of quark gluon plasma (QGP) in collisions of nuclei at relativistic energies. The jet-quenching [6] and elliptic flow [7] have already been confirmed in collision of lead nuclei at $\sqrt{{ }^{s} \mathrm{NN}}=2.76 \mathrm{TeV}$ at the Large Hadron Collider. The simplest measure 


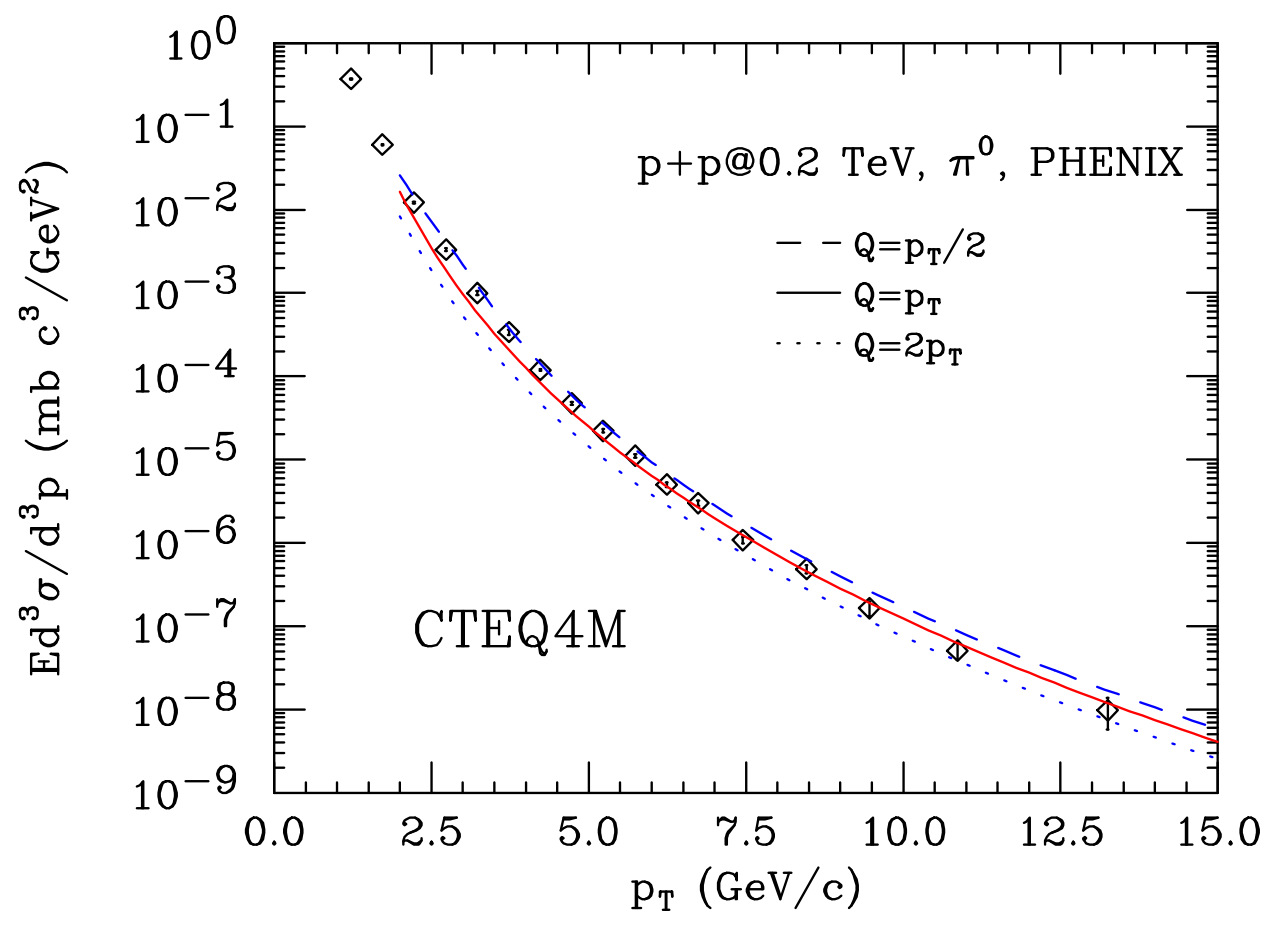

Figure 1. A comparison of inclusive production of neutral pions [1] with NLO pQCD calculations at $\sqrt{{ }^{s} \mathrm{NN}}=200 \mathrm{GeV}$.

of the jet-quenching at large $p_{T}$ is given by the nuclear modification factor $R_{\mathrm{AA}}$, which provides the ratio of particle yield in AA collisions to that in pp, defined as:

$$
R_{\mathrm{AA}}=\frac{\left(1 / N_{\mathrm{evt}}^{\mathrm{AA}}\right) d^{2} N_{\mathrm{AA}} / d^{2} p_{T} d y}{N_{\mathrm{coll}}\left(1 / N_{\mathrm{evt}}^{\mathrm{pp}}\right) d^{2} N_{\mathrm{pp}} / d^{2} p_{T} d y}
$$

where $N_{\text {evt }}$ is the number of events and $N_{\text {coll }}$ is the number of binary (nucleon-nucleon) collisions. In absence of any nuclear modification, $R_{\mathrm{AA}}$ would be unity at large $p_{T}$. The larger density of the medium likely at the higher LHC energies is expected to give rise to a larger loss of energy for a given $p_{T}$, which should lead to a larger suppression. Yet the expectation that the spectrum of the partons at large $p_{T}$ will fall less steeply at the LHC would mean a smaller suppression for the same energy loss of parton of a given $p_{T}$. Thus indeed the $R_{\mathrm{AA}}$ at LHC shows an interesting behaviour: it decreases with increase in $p_{T}$ in the window of $p_{T} \approx 2-5 \mathrm{GeV} / c$ and then rises to reach a value of about 0.4 at $p_{T} \approx 20 \mathrm{GeV} / c$. The results for the most central collisions at RHIC energies can also be interpreted to suggest the emergence of a similar trend. In the present work we suggest that this result is indicative of change of the mechanism for energy loss as $p_{T}$ rises beyond 5-7 GeV/c as the Landau Pomeranchuk Midgal effect sets in to suppress radiation of gluons.

In the next section we give the formulation of our treatment and introduce various mechanisms of energy-loss used in our work. In section III, we discuss our results at RHIC and LHC energies for the 0-5\% most central collisions. Finally we give a short discussion and conclusions. 
Evolution of parton energy loss...

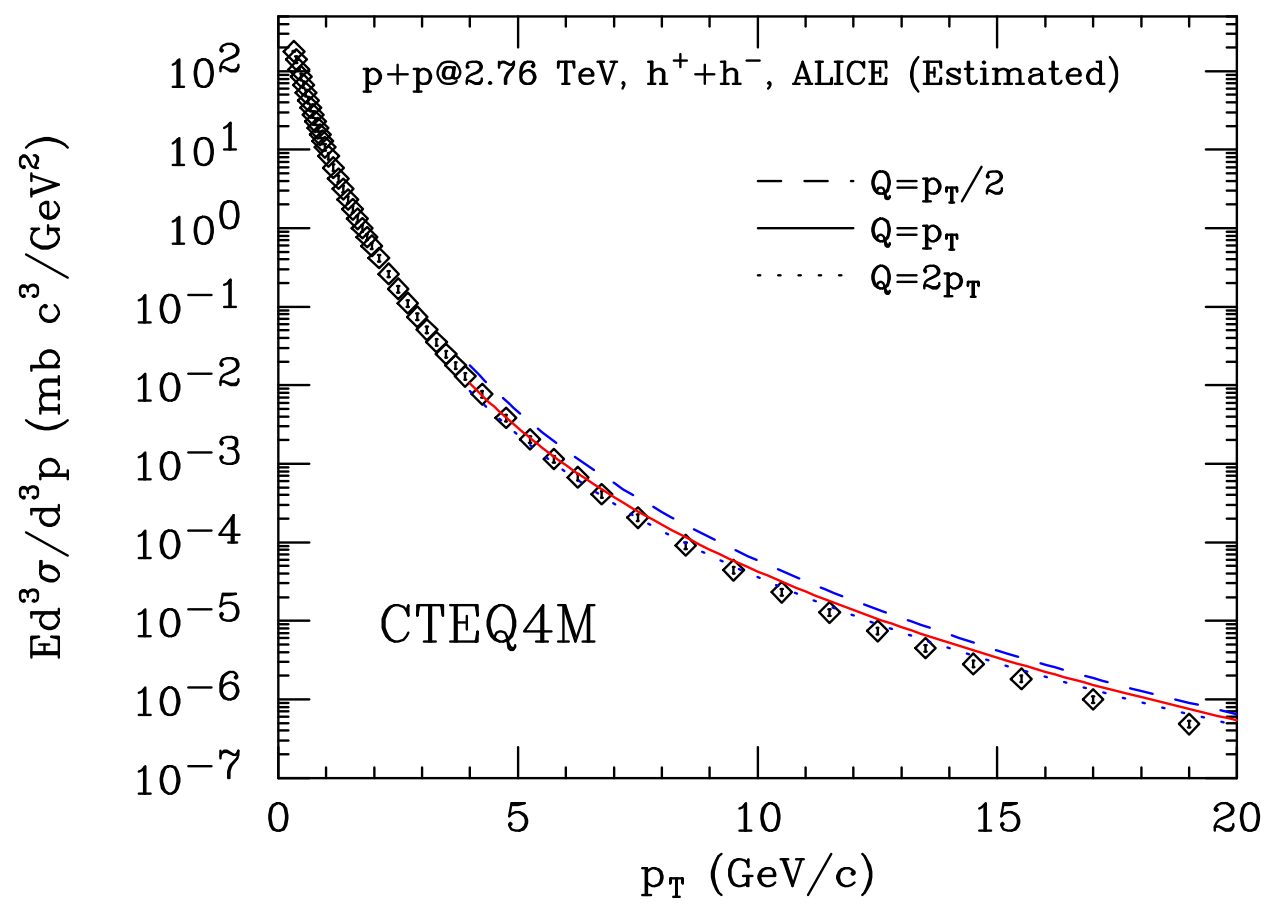

Figure 2. A comparison of inclusive production of charged hadrons estimated by the ALICE collaboration [6] with NLO pQCD calculations at $\sqrt{{ }^{{ }^{\mathrm{NN}}}}=2.76 \mathrm{TeV}$.

\section{Formulation}

\subsection{Particle production in pp collisions}

As a first step we check if the next-to-leading order perturbative QCD [8] describes the $p p$ data used as a reference in the experimental studies. This is important since, for the LHC measurement, the $p p$ data are estimated by using a variety of scaling properties of the particle production in hadronic collisions.

In perturbative QCD, the inclusive cross-section for the production of particle $h$ in a nucleon-nucleon collision is given by:

$$
\begin{array}{r}
E_{h} \frac{d^{3} \sigma}{d^{3} p_{h}}\left(\sqrt{s}, p_{h}\right)=\int d x_{a} \int d x_{b} \int d z \sum_{i, j} F_{i}\left(x_{a}, Q^{2}\right) F_{j}\left(x_{b}, Q^{2}\right) \\
D_{c / h}\left(z, Q_{f}^{2}\right) E_{c} \frac{d^{3} \sigma_{i j \rightarrow c X}}{d^{3} p_{c}},
\end{array}
$$

where $F_{i}\left(x, Q^{2}\right)$ is the distribution function for the $i$-th parton in a nucleon, $D_{c / h}$ is hadron fragmentation function at $z=p_{h} / p_{c}$, and $\sigma_{i j \rightarrow c X}$ is parton-parton cross-section. We include leading order, $\mathcal{O}\left(\alpha_{s}^{2}\right)$ processes, like:

$$
\begin{aligned}
& q+q \rightarrow q+q, \\
& q+\bar{q} \rightarrow q+\bar{q}, \\
& q+g \rightarrow q+g, \\
& g+g \rightarrow g+g,
\end{aligned}
$$


and the next-to-leading order, $\mathcal{O}\left(\alpha_{s}^{3}\right)$, sub-processes such as:

$$
\begin{aligned}
& q+q \rightarrow q+q+g, \\
& q+\bar{q} \rightarrow q+\bar{q}+g, \\
& q+q^{\prime} \rightarrow q+q^{\prime}+g, \\
& q+\bar{q} \rightarrow q^{\prime}+\bar{q}^{\prime}+g, \\
& g+g \rightarrow g+g+g
\end{aligned}
$$

The running coupling constant $\alpha_{s}\left(\mu^{2}\right)$, calculated at next-to-leading order, is given by

$$
\alpha_{s}\left(\mu^{2}\right)=\frac{12 \pi}{\left(33-2 N_{f}\right) \ln \left(\mu^{2} / \Lambda^{2}\right)}\left[1-\frac{6\left(153-19 N_{f}\right) \ln \ln \left(\mu^{2} / \Lambda^{2}\right)}{\left(33-2 N_{f}\right)^{2} \ln \left(\mu^{2} / \Lambda^{2}\right)}\right]
$$

where $\mu$ is the renormalization scale, $N_{f}$ is number of flavours, and $\Lambda=\Lambda_{\mathrm{QCD}}$. We use CTEQ4M structure functions [9] and Binnewies, Kniehl, and Kramer [10] fragmentation functions. We use the factorization, renormalization, and fragmentation scales as $Q=p_{T}$, though we have checked the results for the particle production in $p p$ collisions using scales $Q=p_{T} / 2$ and $Q=2 p_{T}$ as well.

Our results along with the $\pi^{0}$ data measured by the PHENIX experiment [11] are shown in Fig. 1, We see a very good description of the experimental results with-out any free parameters. Similar results have been repeatedly reported earlier (see e.g. Ref. [11]).

For the $p p$ centre of mass energy of $2.76 \mathrm{TeV}$, no experimental data is available and the ALICE collaboration has utilized a multi-pronged approach to estimate [6] this using several well established scaling relations. This estimate and our calculations are shown in Fig. 2, The good agreement of the estimated cross-sections with the NLO pQCD results, holds out the hope of getting an accurate measure of the medium modification of $R_{\mathrm{AA}}$ in such studies.

\subsection{Medium modification of $R_{A A}$}

By now it is very well established that the medium modification of $R_{\mathrm{AA}}$ is mainly caused by the energy-loss suffered by partons while they traverse the plasma due to collisions and radiation of gluons, before they fragment and the nuclear shadowing. The nuclear shadowing accounts for the modification of distribution of quarks and gluons inside nuclei compared to that in free nucleons. We use the nuclear shadowing function obtained by Eskola, Kolhinen, and Salgado [12] which is known to describe the NMC data accurately.

A substantial body of literature exists on the dynamics of propagation of fast partons through the hot and dense medium produced in relativistic heavy ion collisions, where they lose part of their energy through collisions and radiation of gluons before fragmenting into hadrons. 

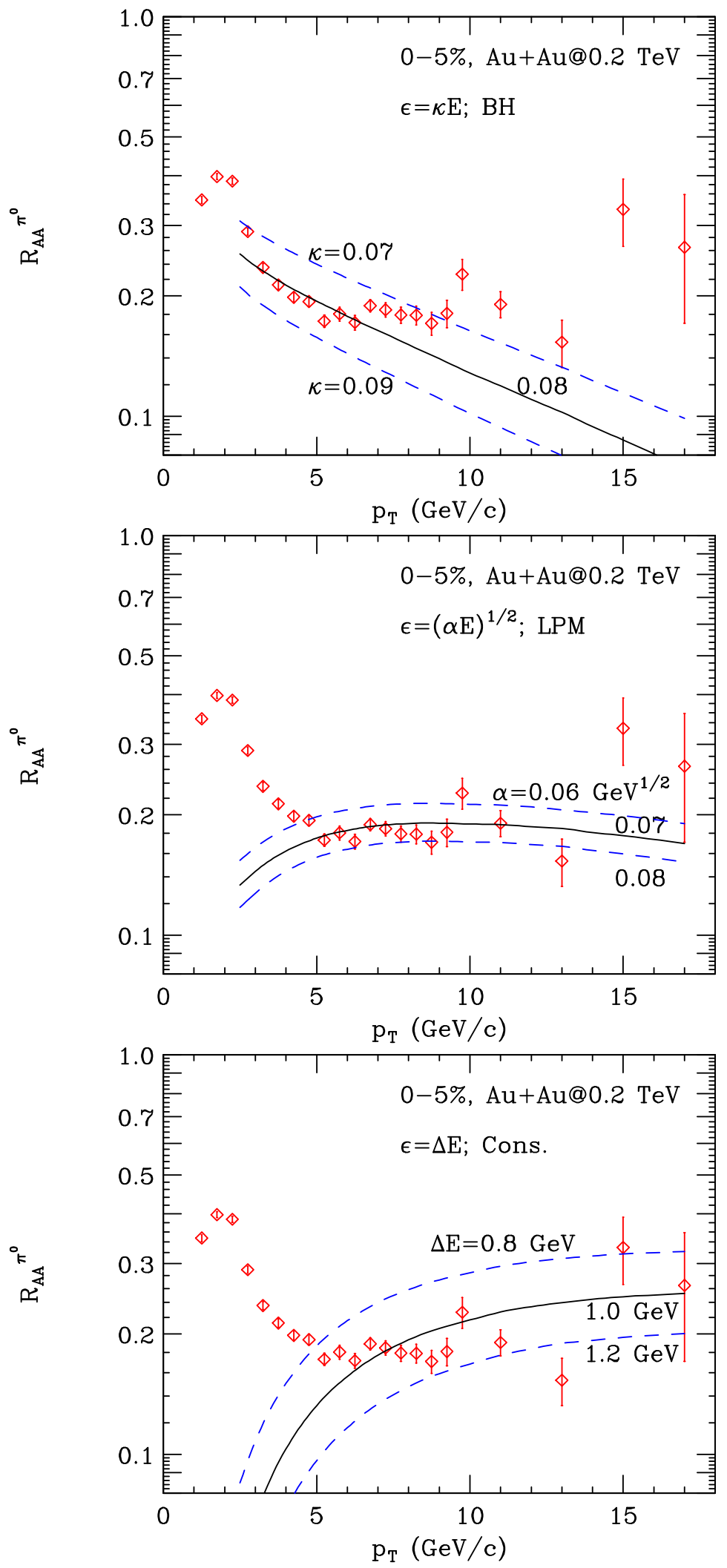

Figure 3. The nuclear modification function for the production of neutral pions for the $0-5 \%$ most central collision of gold nuclei at the top RHIC energy. $\epsilon$ is energy loss per collision and the mean free path is taken as $1 \mathrm{fm}$. The experimental results are from the PHENIX collaboration [27]. 
These developments can be broadly categorized as follows:

- The Higher Twist Approximation developed by Guo and Wang [13, which is based upon the huger twist correction for final state partons in $e+A$ collisions.

- The so-called AMY formalism developed by Arnold, Moore, and Yaffe [14] based on hard thermal loop resummation in a pertubative plasma.

- The so-called ASW formalism developed by Armesto, Salgado, and Wiedemann [15] which resums multiple soft gluon emission in the BDMPS approach [16] (see later) in a finite length medium using Poisson statistics, and

- The GLV formalism developed by Gyulassy, Levai, and Vitev [17] which considers scattering centres in the opacity expansion.

Several developments tracing the evolution of the entire jet in the medium using Monte Carlo jet-quenching modules, e.g. PYQUEN [18], Q-PYTHIA [19], JEWEL [20], YaJEM [21], and MARTINI [22] have also been reported. Many of these developments have been discussed in detailed reviews [23, 24].

We use a simple phenomenological model [25] to study the evolution of the mechanism of energy loss with increasing $p_{T}$ and centre of mass energy. This has been shown to be quite successful in explaining the results for $R_{\mathrm{AA}}$ [26] at RHIC energies. It has also been used extensively to illustrate various consequences of jet-quenching, e.g. photon-tagged jets and di-hadron correlations etc.

The advantage of this treatment, as we shall see, lies in giving reasonable description of the medium modification in terms of energy loss per collision and the mean-free of the parton, which covers an average path length in the medium. We shall see that this works quite well and also helps us to distinguish the energy dependence of the energy loss mechanism.

We focus our attention on the central rapidity and central collisions, so that the parton propagates in the transverse direction and the azimuthal dependence of the pathlength etc. can be safely ignored. Taking the path length as $L$ and the mean free-path of the parton $a$ as $\lambda_{a}$, we estimate the probability for a parton to scatter $n$ times before escaping as

$$
P_{a}(n, L)=\frac{\left(L / \lambda_{a}\right)^{n}}{n !} e^{-L / \lambda_{a}} .
$$

Following Ref. [25], we modify the hadronic fragmentation function $D_{c / h}\left(z, Q^{2}\right)$ to include multiple scattering and the energy loss of the parton in the nuclear medium. Assuming that the average energy loss per collision suffered by the parton $a$ is $\epsilon_{a}$, the nuclear fragmentation function can be written as:

$$
\begin{aligned}
z D_{c / h}\left(z, L, Q^{2}\right)=\frac{1}{C_{N}^{a}} \sum_{n=0}^{N} P_{a}(n, L) & {\left[z_{n}^{a} D_{c / h}^{0}\left(z_{n}^{a}, Q^{2}\right)\right.} \\
& \left.+\sum_{m=1}^{n} z_{m}^{a} D_{g / h}^{0}\left(z_{m}^{a}, Q^{2}\right)\right],
\end{aligned}
$$


where $z_{n}^{a}=z E_{T}^{a} / E_{n}^{a}, E_{n+1}^{a}=E_{n}^{a}-\epsilon_{n}^{a}, z_{m}^{a}=p_{T} / \epsilon_{m}^{a}, n$ is the maximum number of collisions for which $z_{n}^{a} \leq 1, D_{c / h}^{0}$ is the hadronic fragmentation function which gives the probability for the fragmentation of a quark or a gluon to fragment into a $\pi^{0}$ or a charged particle (or any particular hadron in general), and

$$
C_{N}^{a}=\sum_{n=0}^{N} P_{a}(n, L) .
$$

Of-course $E_{0}^{a}=E_{T}^{a}$ in an obvious notation. In the Eq. 7, the first term corresponds to the fragmentation of the leading parton and the second term comes from the emitted gluons having energy $\epsilon_{a}^{m}$. The average number of scatterings over the distance $L$ is $L / \lambda_{a}$, where we take $L=1.2\left(0.5 \times N_{\text {part }}\right)^{1 / 3}$ and $\lambda_{a}=1 \mathrm{fm}$. (This value of $L$ should suffice for the most central collisions considered here. For large impact-parameters $L$ will vary considerably with the azimuthal angle and its average value will be smaller. Consequences of this variation along with those of changing $\epsilon_{a}$ and $\lambda_{a}$ with quarks/gluons will be reported shortly.) As mentioned earlier, we use BKK fragmentation functions from ref. [10] and take the factorization, renormalization, and fragmentation scales to be equal to $p_{T}$.

\subsection{Parton energy loss}

We employ three different prescriptions for the the energy loss per collision $\left(\epsilon_{a}=\right.$ $\left.\lambda_{a} \times d E_{a} / d x\right)$. The choices are inspired by the excellent discussion in Baier et. al [16]. These authors argue that for light quarks and gluons the collisional energy loss $(d E / d x)$ is quite small $\left(0.2-0.3 \mathrm{GeV} / \mathrm{fm}\right.$ for quarks and $\frac{9}{4}$ times this value for gluons at $T \approx 250$ $\mathrm{MeV}$ ). Thus the energy loss for them is dominated by the mechanism of radiation of gluons.

The radiation of gluons is then best discussed in terms of the formation time of the radiated gluon:

$$
t_{\text {form }} \approx \frac{\omega}{k_{T}^{2}}
$$

where $\omega \gg k_{T}$ is the energy of the gluon and $k_{T}$ is the transverse momentum of the gluon. For small $\omega\left(t_{\text {form }} \leq \lambda\right)$ incoherent radiation takes place over $L / \lambda$ scattering centres. This is the so-called Bethe-Heitler regime and one can derive:

$$
-\frac{d E}{d x} \approx \frac{\alpha_{s}}{\pi} N_{c} \frac{1}{\lambda} E
$$

where $N_{c}=3$. We shall write $\epsilon \approx \kappa E$ for this case and determine $\kappa$ using the $R_{\mathrm{AA}}$ measured in the experiment.

When the formation time $t_{\text {form }}$ is greater than $\lambda$ but less than $L, N_{\text {coh }}$ scattering centres act as a single source of radiation, where

$$
N_{\mathrm{coh}}=\ell_{\mathrm{coh}} / \lambda
$$

and

$$
\ell_{\mathrm{coh}} \approx \frac{\omega}{\left.<k_{T}^{2}\right\rangle_{\mathrm{coh}}}
$$



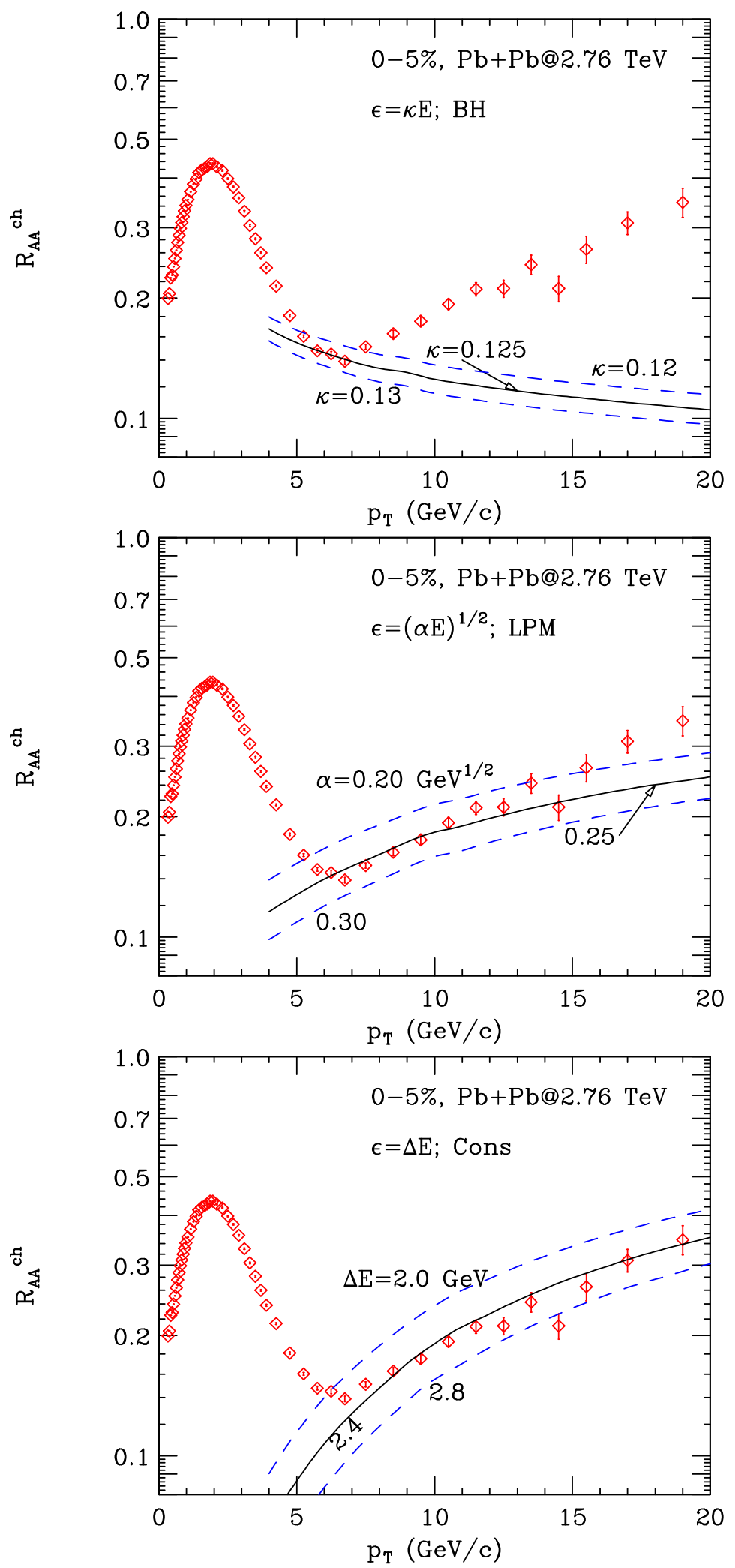

Figure 4. The nuclear modification function for the production of charged particles for the $0-5 \%$ most central collision of lead nuclei at $\sqrt{{ }^{s} \mathrm{NN}}=2.76 \mathrm{TeV}$ measured at the Large Hadron Collider. $\epsilon$ is energy loss per collision and the mean free path is taken as $1 \mathrm{fm}$. The experimental data are from the ALICE experiment [6]. 
Evolution of parton energy loss...
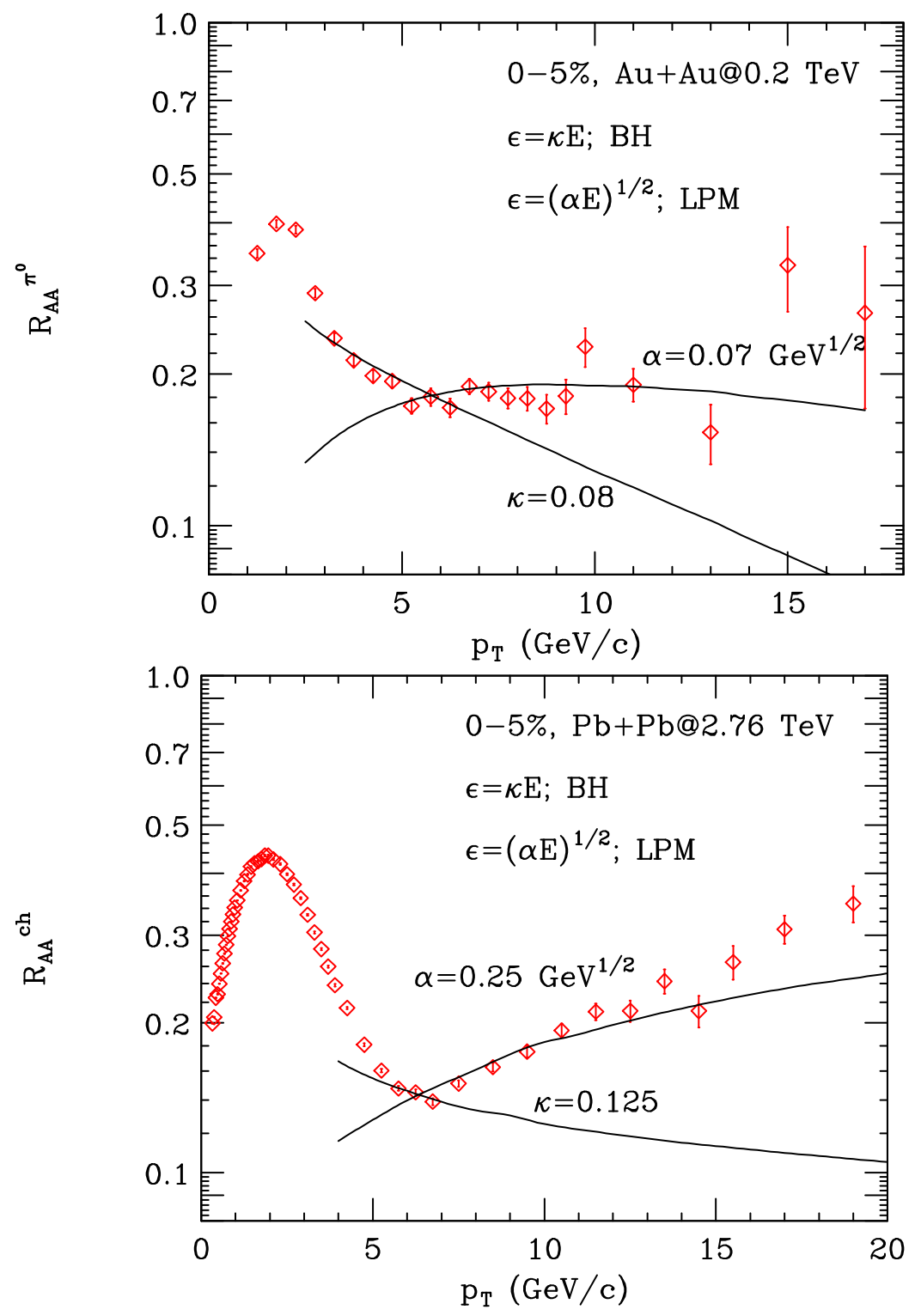

Figure 5. Evolution of the mechanism of energy loss from Bethe Heitler to Landau Pomeranchuk Midgal as a function of $p_{T}$ at RHIC and LHC.

where $\left\langle k_{T}^{2}>_{\text {coh }}\right.$ is the accumulated transverse momentum and $\approx N_{\text {coh }} \times<k_{T}^{2}>$. Thus one can write:

$$
N_{\mathrm{coh}} \approx \sqrt{\frac{\omega}{\lambda<k_{T}^{2}>}}=\sqrt{\frac{\omega}{E_{\mathrm{LPM}}}}
$$

where $E_{\mathrm{LPM}}=\lambda<k_{T}^{2}>$ is the energy which separates the $\mathrm{BH}$ regime from the LPM regime. Now, one can write:

$$
-\frac{d E}{d x} \approx \frac{\alpha_{s}}{\pi} \frac{N_{c}}{\lambda} \sqrt{E_{\mathrm{LPM}} E}
$$

In the present work, we denote this scenario with $\epsilon=\sqrt{\alpha E}$ and determine $\alpha$ from a description of $R_{\mathrm{AA}}$. 


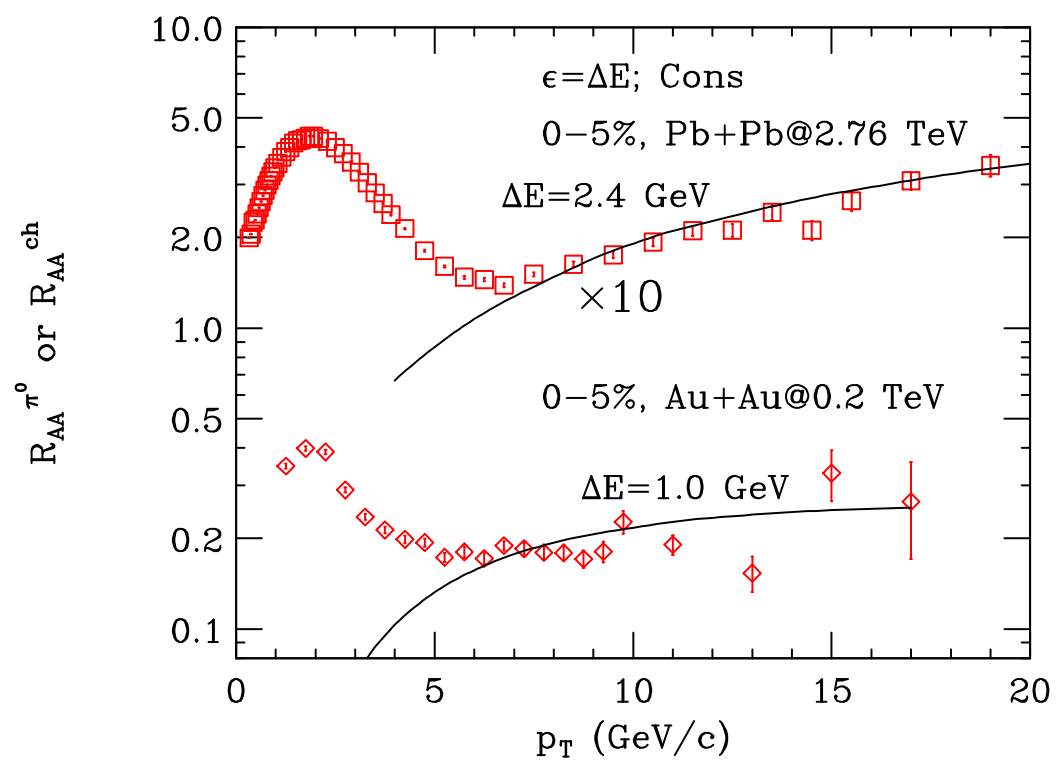

Figure 6. Doubling of energy loss per collision as $\sqrt{{ }^{s} \mathrm{NN}}$ is increased from $200 \mathrm{GeV}$ to $2.76 \mathrm{TeV}$.

Finally, when the formation time is much larger than the path-length $L$ the coherence is complete and the medium induced total energy loss becomes proportional to the square of $L$, and one gets:

$$
-\frac{d E}{d x}=\frac{\alpha_{s}}{\pi} N_{c} \frac{<k_{T}^{2}>}{\lambda} L .
$$

We shall denote this case by having $\epsilon=$ constant, for a given $L$. One may need to check it for cases having varying $L$ (e.g., centrality dependence of $R_{\mathrm{AA}}$ ). Results for such a study would be published shortly. The NLO code of Aurenche et al had earlier been modified by Jeon et al [26] to incorporate the medium modified fragmentation functions. We use it for the calculations reported here.

\section{Results}

We have already seen that the NLO pQCD calculations accurately describe the results for particle production in $p p$ collisions at both the energies under consideration (See Figs. (1,2).

The nuclear modification factor $R_{\mathrm{AA}}$ obtained in the present work for central collision of gold nuclei at $\sqrt{{ }^{\mathrm{NN}}}$ equal to $200 \mathrm{GeV}$, using the three energy loss mechanisms discussed above are shown in Fig. 3. The so-called Bethe Heitler mechanism is seen to accurately describe the nuclear modification for $2.5<p_{T}<6 \mathrm{GeV} / c$ (top panel).

It is interesting to note that the condition necessary for the multiple scatterings to be incoherent, when the mean free path is about $1 \mathrm{fm}$ requires that the formation time of the gluon is less than $1 \mathrm{fm} / c$. This will be satisfied if the energy of the radiated gluon 
is less than $5 \mathrm{GeV}$ and $<k_{T}^{2}>\approx 1(\mathrm{GeV} / \mathrm{c})^{2}$. Thus indeed the partons having energy up to $5-7 \mathrm{GeV}$ would populate this $p_{T}$ window. The value of $\kappa \approx 8 \%$, obtained here is close to (but higher than) that obtained by Jeon et al. [26] for $0-10 \%$ centrality. However it is smaller than the value suggested by the Eq. 10. We shall discuss this later.

The nuclear modification shows a clear change in slope around $p_{T}$ equal to $5 \mathrm{GeV} / c$. We also note that $E_{\mathrm{LPM}} \approx 5 \mathrm{GeV}$ as discussed above. Thus it is interesting that the energy loss implied by Eq. 14 provides an accurate description of $R_{\mathrm{AA}}$ for $5 \mathrm{GeV} / c$ $<p_{T}<12 \mathrm{GeV} / c$, when we take $\alpha$ as $0.07 \mathrm{GeV}^{1 / 2}$ (Fig. 3, middle panel). We again see that while the form of the energy loss is given by Eq. 14, the coefficient for the energy loss we get is about half as small.

The statistics for the modification factor is quite poor at larger $p_{T}$, however the average behaviour seen (see Fig. 3, lower panel) is in fair agreement with energy loss mechanism given by Eq. 15, though once again the energy loss per collision is smaller than that implied by this equation.

The results for $\sqrt{{ }^{s} \mathrm{NN}}=2.76 \mathrm{TeV}$, (see Fig. 4) are also quite interesting. We now see (top panel) that the $\mathrm{BH}$ mechanism provides a description of the data over a limited $p_{T}$ window of $5-7 \mathrm{GeV} / c$. It is quite likely that at these higher energies the validity of hydrodynamics may extend to higher $p_{T}$ than that witnessed at RHIC energies and thus the radial flow may affect the $p_{T}$ window up to $5 \mathrm{GeV} / c$ as well as our results.

The so-named LPM regime is seen to hold over the $p_{T}$ window of $7-14 \mathrm{GeV} / c$ (see Fig, 4, middle panel) with $\alpha \approx 0.25 \mathrm{GeV}^{1 / 2}$, which is still smaller (by about $30 \%$ ) than that implied by Eq. 14,

The mechanism which gives a constant energy loss per collision is seen to accurately describe the data over the largest $p_{T}$ window of $10-20 \mathrm{GeV} / c$ (see Fig, lower panel). This is interesting as this mechanism is supposed to work for $L<L_{\mathrm{cr}}$ where $L_{\mathrm{cr}}=\lambda \sqrt{E / E_{\mathrm{LPM}}}$ which should be true for $E \geq$ about $125 \mathrm{GeV}$ for $L=5 \mathrm{fm}$, while it seems to be operating even at lower energies. The energy loss per collision is still smaller than that implied by Eq. 15, though it is about twice as large as at the top RHIC energy.

In order to bring out the changing mechanism for energy loss with increasing $p_{T}$ we now plot the final results for RHIC and LHC energies using the BH and LPM mechanisms (see Fig. [5, upper and lower panels). The results at RHIC energies show a dramatic change in the mechanism around $p_{T}$ of $5 \mathrm{GeV} / c$, as indeed one expects from the consideration of formation time of the radiated gluons and the mean free path of the partons.

The comparison for the constant energy loss per collision for the RHIC and the LHC energies is shown in Fig. 6. We see that while the results at RHIC are only indicative of possible emergence of this mechanism at larger $p_{T}$, the results at LHC clearly favour this mechanism for $p_{T}>10 \mathrm{GeV} / c$. 


\section{Summary and Discussions}

We have adopted a simple and transparent model of energy loss of partons traversing the medium created in relativistic collision of heavy nuclei. The fragmentation functions of the partons are then modified to account for the energy loss (and also fragmentation of radiated gluons) and nuclear modification function $R_{\mathrm{AA}}$ calculated. The energy loss per collision is taken to be either proportional to the energy of the parton, square-root of the energy of the parton, or a constant, inspired by the treatment of energy loss depending on the formation time of the radiated gluon and the mean free path of the parton. The reference particle spectra are calculated using NLO pQCD.

A dramatic change in the energy loss mechanism is seen at $p_{T} \approx 5 \mathrm{GeV} / c$. We find that the Bethe- Heitler mechanism of incoherent scattering prevails for $p_{T} \leq 5 \mathrm{GeV} / c$ at both RHIC and LHC energies, while the so-called LPM mechanism, which gives energy loss per collision as proportional to the square-root of the energy prevails over the $p_{T}$ window of $5-15 \mathrm{GeV} / c$. In an interesting observation we find that the constant energy loss per collision suggestive of a total energy loss increasing with the square of the path length may already be becoming relevant for $p_{T}>10 \mathrm{GeV} / c$. This mechanism is seen to prevail over the $p_{T}$ window of $10-20 \mathrm{GeV} / c$ at $\sqrt{{ }^{s} \mathrm{NN}}=2.76 \mathrm{TeV}$.

While the results generally follow the qualitative arguments given in Ref. [16] for the form of the energy-loss per collision as the energy of the parton changes, the energy-loss coefficients are smaller than those implied in the above work. One obvious difference is while the medium produced in these collisions is expanding and cooling the treatment of Ref. [16] is basically for a static medium (with static centres of scattering, a la Glauber model), though modifications for expansion are available. Obviously, in an expanding medium the parton will initially move in a hotter layer and later in cooler layers. The energy loss per collision emerging in the present work will then give an average over the history of evolution. The expansion of the plasma will also lead to a $L$ larger than the radius of the system, at least at LHC energies. However the expanding volume beyond the volume of the plasma produced originally will also be cooler and thus the energy loss there may be small. It is also likely that the partons produced near the centre would have already lost most of their energy by the time they enter this volume, and thus they would fragment to low energy hadrons. The boundaries of the different mechanisms may also not be quite sharp.

It will be interesting to see how these mechanisms evolve with the centrality of the collision [28] and how do they fare when the dynamics of evolution is accounted for [29]. Is this simple but transparent treatment able to account for the azimuthal anisotropy of the momentum distribution for non-central collisions at large $P_{T}$ ? These will be addressed in a forthcoming publication [30]. We add that, even though the discussion of Ref. [16] focuses on radiative energy loss of the partons the treatment in terms of energy loss per collision could easily accommodate the collisional energy loss, as long as it is small.

We conclude that the mechanism of energy loss of the partons weaves a rich tapestry 
Evolution of parton energy loss...

depending on the energy of the parton and the properties of the medium. The simple model of multiple scattering used in the work is able to reveal the evolution of the mechanism of energy loss with $p_{T}$.

\section{Acknowledgments}

Valuable discussions with Somnath De, Umme Jamil, Sangyong Jeon, and Mohammed Younus are gratefully acknowledged.

\section{References}

[1] X. N. Wang, Phys. Rev. C 63, 054902 (2001); M. Gyulassy, I. Vitev, X. N. Wang, Phys. Rev. Lett. 86, 2537 (2001).

[2] K. Adcox et al. [PHENIX Collaboration], Phys. Rev. Lett. 88, 022301 (2002); J. Adams et al. [STAR Collaboration], Phys. Rev. Lett. 91, 172302 (2003).

[3] S. S. Adler et al. [PHENIX Collaboration], Phys. Rev. Lett. 91, 182301 (2003); S. Esumi (for the PHENIX collaboration), Nucl. Phys. A715, 599 (2003); C. Adler et al. [STAR Collaboration], Phys. Rev. Lett. 90, 032301 (2003); ibid. 89132301 (2002); ibid. 87182301 (2001).

[4] P. Huovinen, P. F. Kolb, U. W. Heinz, P. V. Ruuskanen and S. A. Voloshin, Phys. Lett. B 503, 58 (2001); D. Teaney, J. Lauret and E. V. Shuryak, arXiv:nucl-th/0110037.

[5] R. J. Fries, B. Muller, C. Nonaka and S. A. Bass, Phys. Rev. Lett. 90, 202303 (2003) arXiv:nucl-th/0301087, R. J. Fries, B. Muller, C. Nonaka and S. A. Bass, Phys. Rev. C 68, 044902 (2003) arXiv:nucl-th/0306027, V. Greco, C. M. Ko and P. Levai, Phys. Rev. Lett. 90, 202302 (2003) arXiv:nucl-th/0301093.

[6] K. Aamodt et al. [ALICE Collaboration], Phys. Lett. B 696, 30 (2011) arXiv:1012.1004 [nucl-ex]].

[7] K. Aamodt et al. [The ALICE Collaboration], arXiv:1011.3914 [nucl-ex].

[8] P. Aurenche, M. Fontannaz, J. P. Guillet, B. A. Kniehl and M. Werlen, Eur. Phys. J. C 13, 347 (2000), F. Aversa, M. Greco, P. Chiappetta and J. P. Guillet, Z. Phys. C 46, 253 (1990), F. Aversa, M. Greco, P. Chiappetta and J. P. Guillet, Phys. Rev. Lett. 65, 401 (1990), R. K. Ellis and J. C. Sexton, Nucl. Phys. B 269, 445 (1986).

[9] H.L. Lai et al., Phys. Rev. D 55, 1280 (1997).

[10] J. Binnewies, B. A. Kniehl and G. Kramer, Phys. Rev. D 52, 4947 (1995) arXiv:hep-ph/9503464, J. Binnewies, B. A. Kniehl and G. Kramer, Z. Phys. C 65, 471 (1995) arXiv:hep-ph/9407347).

[11] A. Adare et al. [PHENIX Collaboration], Phys. Rev. D 76, 051106 (2007) arXiv:0704.3599 [hep-ex]], S. S. Adler et al. [PHENIX Collaboration], Phys. Rev. Lett. 91, 241803 (2003) arXiv:hep-ex/0304038.

[12] K. J. Eskola, V. J. Kolhinen, and C. A. Salgado, Eur. Phys. J. C 9, 61 (1999), JYFL-8/98, USFT/14-98, hep-ph/9807297; K. J. Eskola, V. J. Kolhinen, and P. V. Ruuskanen, Nucl. Phys. B 535, 351 (1998), CERN-TH/97-345, JYFL-2/98, hep-ph/9802350.

[13] X. f. Guo and X. N. Wang, Phys. Rev. Lett. 85, 3591 (2000) arXiv:hep-ph/0005044, X. N. Wang and X. f. Guo, Nucl. Phys. A 696, 788 (2001) arXiv:hep-ph/0102230.

[14] P. B. Arnold, G. D. Moore and L. G. Yaffe, JHEP 0206, 030 (2002) arXiv:hep-ph/0204343, S. Jeon and G. D. Moore, Phys. Rev. C 71, 034901 (2005) arXiv:hep-ph/0309332.

[15] C. A. Salgado and U. A. Wiedemann, Phys. Rev. Lett. 89, 092303 (2002) arXiv:hep-ph/0204221], C. A. Salgado and U. A. Wiedemann, Nucl. Phys. A 715, 783 (2003) arXiv:hep-ph/0209025, C. A. Salgado and U. A. Wiedemann, Phys. Rev. D 68, 014008 (2003) arXiv:hep-ph/0302184].

[16] R. Baier, D. Schiff and B. G. Zakharov, Ann. Rev. Nucl. Part. Sci. 50, 37 (2000) arXiv:hep-ph/0002198, R. Baier, Y. L. Dokshitzer, A. H. Mueller, S. Peigne and D. Schiff, Nucl. Phys. B 484, 265 (1997) arXiv:hep-ph/9608322. R. Baier, Y. L. Dokshitzer, 
A. H. Mueller, S. Peigne and D. Schiff, Nucl. Phys. B 483, 291 (1997) arXiv:hep-ph/9607355,

R. Baier, Y. L. Dokshitzer, A. H. Mueller and D. Schiff, Phys. Rev. C 58, 1706 (1998) arXiv:hep-ph/9803473.

[17] M. Gyulassy, P. Levai and I. Vitev, Nucl. Phys. B 571, 197 (2000) arXiv:hep-ph/9907461,. M. Gyulassy, P. Levai and I. Vitev, Phys. Rev. Lett. 85, 5535 (2000) arXiv:nucl-th/0005032, M. Gyulassy, P. Levai and I. Vitev, Nucl. Phys. B 594, 371 (2001) arXiv:nucl-th/0006010.

[18] I. P. Lokhtin and A. M. Snigirev, Eur. Phys. J. C 45, 211 (2006) arXiv:hep-ph/0506189.

[19] N. Armesto, L. Cunqueiro and C. A. Salgado, Eur. Phys. J. C 63, 679 (2009) arXiv:0907.1014 [hep-ph]].

[20] K. Zapp, J. Stachel and U. A. Wiedemann, Phys. Rev. Lett. 103, 152302 (2009) arXiv:0812.3888 [hep-ph]], K. Zapp, G. Ingelman, J. Rathsman, J. Stachel and U. A. Wiedemann, Eur. Phys. J. C 60, 617 (2009) arXiv:0804.3568 [hep-ph]].

[21] T. Renk, Phys. Rev. C 78, 034908 (2008) arXiv:0806.0305 [hep-ph]].

[22] B. Schenke, C. Gale and S. Jeon, Acta Phys. Polon. Supp. 3, 765 (2010) [arXiv:0911.4470 [hep-ph]], B. Schenke, C. Gale and S. Jeon, Phys. Rev. C 80, 054913 (2009) [arXiv:0909.2037 [hep-ph]].

[23] R. J. Fries and C. Nonaka, arXiv:1012.1881 [nucl-th].

[24] A. Majumder and M. Van Leeuwen, arXiv:1002.2206 [hep-ph].

[25] X. N. Wang, Z. Huang and I. Sarcevic, Phys. Rev. Lett. 77, 231 (1996) arXiv:hep-ph/9605213, X. N. Wang and Z. Huang, Phys. Rev. C 55, 3047 (1997) arXiv:hep-ph/9701227.

[26] S. Jeon, J. Jalilian-Marian and I. Sarcevic, Nucl. Phys. A 715, 795 (2003) arXiv:nucl-th/0211084, S. Jeon, J. Jalilian-Marian and I. Sarcevic, Phys. Lett. B 562, 45 (2003) arXiv:nucl-th/0208012, S. Jeon, J. Jalilian-Marian and I. Sarcevic, Nucl. Phys. A 723, 467 (2003) arXiv:hep-ph/0207120.

[27] A. Adare et al. [PHENIX Collaboration], Phys. Rev. Lett. 101, 232301 (2008) arXiv:0801.4020 [nucl-ex]].

[28] A. Adare et al. [PHENIX Collaboration], Phys. Rev. Lett. 105, 142301 (2010) arXiv:1006.3740 [nucl-ex]].

[29] S. A. Bass, C. Gale, A. Majumder, C. Nonaka, G. Y. Qin, T. Renk and J. Ruppert, Phys. Rev. C 79, 024901 (2009) arXiv:0808.0908 [nucl-th]].

[30] Somnath De and D. K. Srivastava, to be published. 